\section{Estrutura dimensional da versão brasileira da Escala de Satisfação com o Processo Interpessoal de Cuidados Médicos Gerais}

\author{
Dimensional structure of the Brazilian version of the \\ Scale of Satisfaction with Interpersonal Processes \\ of General Medical Care
}

\author{
1 Escola Nacional de Saúde \\ Pública Sergio Arouca, \\ Fundação Oswaldo Cruz, Rio \\ de Janeiro, Brasil. \\ 2 Hospital Geral de Nova \\ Iguaçu, Nova Iguaçu, Brasil. \\ 3 Instituto de Medicina \\ Social, Universidade do \\ Estado do Rio de Janeiro, Rio \\ de Janeiro, Brasil. \\ Correspondência \\ M. I. Nascimento \\ Escola Nacional de Saúde \\ Pública Sergio Arouca, \\ Fundação Oswaldo Cruz. \\ Rua Leopoldo Bulhões 1480 , \\ Rio de Janeiro, RJ 21041-210 \\ Brasil. \\ ysamaria@uol.com.br
}

\section{Abstract}

The objective of this study was to reassess the dimensional structure of a Brazilian version of the Scale of Satisfaction with Interpersonal Processes of General Medical Care, proposed originally as a one-dimensional instrument. Strict confirmatory factor analysis (CFA) and exploratory factor analysis modeled within a CFA framework (E/CFA) were used to identify the best model. An initial CFA rejected the one-dimensional structure, while an E/CFA suggested a two-dimensional structure. The latter structure was followed by a new CFA, which showed that the model without cross-loading was the most parsimonious, with adequate fit indices $(C F I=0.982$ and $T L I=$ 0.988), except for RMSEA (0.062). Although the model achieved convergent validity, discriminant validity was questionable, with the square-root of the mean variance extracted from dimension 1 estimates falling below the respective factor correlation. According to these results, there is not sufficient evidence to recommend the immediate use of the instrument, and further studies are needed for a more in-depth analysis of the postulated structures.

Physician-Patient Relations; Questionnaires; Validation Studies; Statistical Factor Analysis
Maria Isabel do Nascimento 1,2

Michael Eduardo Reichenheim 3

Gina Torres Rego Monteiro 1

\section{Introdução}

A relação médico-paciente é um fenômeno complexo, multifatorial, relacionado à satisfação do paciente e à continuidade do cuidado em saúde $1,2,3$. Ela comporta alguns elementos como segurança, honestidade, confiança, reconhecimento e respeito que são essenciais para a sua construção e manutenção. Por vezes, esses atributos são apreendidos em circunstâncias particulares de consultas médicas e que acabam por influenciar o fato de o paciente poder contar com uma fonte sólida para cuidados de saúde 4 .

Alguns autores sinalizam a importância de incorporar medidas de percepções captadas pelo paciente em questionários, quando se pretende fazer um julgamento da relação médico-paciente 5,6. Rosenthal \& Shannon 6 assinalam que a opção por essas medidas tem como vantagens: a facilidade de se obter a informação com rapidez, logo após o paciente utilizar um serviço de interesse; o baixo custo de operacionalização; e a possibilidade de efetuar a coleta independente da completude de prontuários médicos que raramente contém informações sobre aspectos interpessoais.

Considerando o processo interpessoal um construto definido como os aspectos psicossociais da relação médico-paciente que podem afetar os processos técnicos e os resultados em saúde, Stewart et al. 7 desenvolveram um modelo 
conceitual denominado Interpersonal Processes of Care Scale (IPC Scale) contemplando as dimensões "comunicação", "estilo interpessoal” e "participação em tomadas de decisão". Alguns estudos têm fornecido evidências a respeito da seleção e fraseamento dos itens 8 e da definição das três dimensões da IPC Scale 7,9. A IPC Scale 7 valoriza a percepção do paciente em relação à atenção recebida e abarca questões que podem ser alvo de avaliação de qualidade e de melhoramentos, na ótica da gerência de sistemas de serviços de saúde.

A comunicação constitui uma ação comportamental essencial à interação médico-paciente, compreendendo, além da troca de informações, os gestos e as emoções como componentes não verbais da relação, enquanto com o estilo interpessoal se enfatiza o respeito como a base de todas as interações humanas ${ }^{10}$. A participação na tomada de decisão, conduzida à luz das preferências do paciente, leva em conta o desejo de se envolver ativamente nas decisões ou não, bem como a aceitabilidade e/ou habilidade para aderir a determinadas recomendações 7 .

A revisão de instrumentos derivados da IPC Scale 7 revelou que o Prenatal Interpersonal Processes of Care (PIPC) tinha sido adaptado para inquirir sobre a qualidade do pré-natal 11. Ele é multidimensional composto de trinta itens os quais envolvem a interação da paciente com outros profissionais, além dos médicos. Um outro subconjunto de vinte itens do Interpersonal Processes of Care in Diverse Populations Questionnaire (IPCDPQ) 12 foi adaptado com o propósito de verificar se as pessoas com pouca instrução apresentavam, além das dificuldades de compreender as instruções escritas, problemas com a comunicação experimentada nas consultas com os médicos.

Baseados na IPC Scale 7, Somkin et al. 13 propuseram uma escala para aferir a satisfação de usuárias com o processo interpessoal no contexto de cuidados médicos gerais que, para fins de apresentação, será doravante denominada Escala de Satisfação com o Processo Interpessoal de Cuidados Médicos Gerais ou pelo acrônimo ESPICMG. O interesse era capturar, pela frequência dos eventos, um grau de satisfação da paciente quantificado a partir de percepções que resultam da relação interpessoal vivenciadas em consultas com os médicos.

Especificamente, os itens do ESPICMG 13 abordam aspectos de comunicação ou troca de informação entre médicos e pacientes, bem como questões relativas a respeito e discriminação, perguntando a frequência com que os médicos (i) informam a paciente sobre sua própria saúde (item 1); (ii) disponibilizam tempo suficiente para perguntas (item 2); (iii) escutam com paciência o que a paciente tem a dizer (item 3); (iv) perguntam se há alguma dúvida (item 4); (v) tratam a paciente com dignidade (item 5); (vi) demonstram interesse pela paciente (item 6); (vii) aceitam as ideias que a própria paciente tem sobre seus problemas de saúde (item 7); e (viii) demonstram discriminação para com a paciente (item 8).

O ESPICMG foi concebido originalmente em inglês e usado para medir o efeito da satisfação com o processo interpessoal na adesão regular ao rastreamento de câncer feito pelo teste de Papanicolaou e pela mamografia 13 . O conjunto contempla alguns determinantes de satisfação também identificados entre os usuários do sistema público de saúde do Brasil 14. Diante da ausência de qualquer versão em português, ele foi escolhido para adequação ao contexto brasileiro 15 , sendo adotado o roteiro de adaptação transcultural proposto por Herdman et al. 16.

O histórico psicométrico da escala revela apenas uma análise fatorial exploratória utilizando rotação varimax, que evidencia cargas fatoriais acima de 0,6 para todos os itens componentes e uma consistência interna de $\alpha=0,88$ 13. Essa última avaliação psicométrica, bem como o fato de os autores subsequentemente utilizarem a escala na forma de um escore único decorrente do somatório dos itens componentes, sugere que a escala foi tacitamente assumida como sendo unidimensional. No entanto, o modelo conceitual de onde esta deriva não parece sugerir o mesmo. A falta de sintonia sugerida pelo histórico resgatado é evidente. Vale ressaltar a importância da questão dimensional, pois essa estrutura indica a forma como o instrumento deve ser concretamente operacionalizado. A utilização de um escore global somente se justifica se uma escala é efetivamente unidimensional. Caso contrário, usar subescalas separadamente pode ser a melhor forma de acomodar diferentes dimensões de um construto. Claramente, diferentes formas de operacionalização implicam diferentes resultados que, em última instância, prejudicam e ofuscam comparações entre estudos 16 .

O presente estudo está sendo desenvolvido no contexto da prevenção e controle do câncer do colo do útero. O Ministério da Saúde do Brasil tem investido em melhorias do programa de rastreamento do câncer do colo do útero e tem enfatizado a necessidade de tentar aumentar a captação de mulheres que nunca fizeram o teste de Papanicolaou e de melhorar o seguimento daquelas que já realizaram o exame 17. Acredita-se que as mulheres mais satisfeitas com o processo interpessoal também tenham maior propensão para aderir às recomendações de rastreamen- 
to preconizadas. Assim, torna-se importante a realização de estudos para verificar se existem problemas no âmbito dessa relação que possam interferir em tal propósito, utilizando instrumentos válidos e confiáveis. Na falta de um instrumento validado para uso no Brasil com enfoque nos atributos do processo interpessoal, a adaptação de outro concebido em cultura diferente da nossa se fez necessária. Nesse sentido, o presente trabalho tem como objetivo reavaliar a estrutura dimensional da ESPICMG, agora utilizando um ferramental metodológico abrangente e apurado, mais apropriado para dirimir as possíveis inconsistências pendentes.

\section{Material e método}

O presente trabalho foi desenvolvido no Hospital Geral de Nova Iguaçu, unidade integrante do Sistema Único de Saúde (SUS), sob a coordenação da Escola Nacional de Saúde Pública Sergio Arouca, Fundação Oswaldo Cruz (ENSP/ Fiocruz). A unidade é referência para atendimento secundário de lesões precursoras do câncer do colo do útero rastreadas em mulheres residentes na Baixada Fluminense pelo programa de prevenção e controle dessa neoplasia.

\section{População alvo e tamanho da amostra}

A população da pesquisa é formada por mulheres alvo do programa brasileiro de controle do câncer do colo do útero 17 que residem na Baixada Fluminense.

O cálculo do tamanho da amostra foi baseado no número de parâmetros do modelo de mensuração em avaliação, estimado inicialmente em 16 (oito itens e oito erros de mensuração) e na razão de 20 entrevistas por parâmetro 18 , sendo necessário realizar pelo menos 320 entrevistas.

\section{Critérios de inclusão e exclusão}

As participantes foram aleatoriamente selecionadas entre mulheres que visitavam ou acompanhavam pacientes internados no Hospital Geral de Nova Iguaçu. A coleta dos dados foi realizada de outubro de 2008 a dezembro de 2009. Os critérios de inclusão foram a idade (18 a 69 anos) e tempo de residência na Baixada Fluminense (pelo menos 36 meses). Os critérios de exclusão foram problemas de saúde mental impeditivos de responder as perguntas do questionário e estar na unidade, naquele momento, em busca de assistência para a própria saúde.

\section{Seleção da amostra}

A seleção das mulheres foi feita por um dos autores (M.I.N.) em dias alternados, uma hora antes do início do horário reservado à visita dos pacientes internados, na fila de espera para acesso às enfermarias. Como não se dispunha de uma lista diária com o nome dos visitantes, a estratégia de seleção foi baseada na ordem de chegada. À medida que as pessoas iam chegando, a pesquisadora convidava uma a cada cinco mulheres da fila, após breve triagem para avaliar os critérios de inclusão/exclusão do estudo. Caso a convidada declinasse em participar, convidavase a próxima visitante da fila, com os mesmos critérios de triagem. A cada dia, o número de entrevistas realizadas era dependente da existência e do tamanho da fila, da participação de uma ou duas entrevistadoras e do tempo gasto com os procedimentos de seleção e coleta dos dados (aplicação do questionário). Em média, foram realizadas três a seis entrevistas por dia. O trabalho tinha duração de uma hora, encerrando com o início do horário da visita.

Foram convidadas 490 mulheres, tendo sido efetivamente entrevistadas 479 , das quais somente sete não puderam ser incluídas neste estudo por incompletude das informações no instrumento em avaliação. As 472 entrevistas foram realizadas em local reservado, na presença apenas da entrevistadora, de modo a garantir o anonimato e a confidencialidade da informação coletada.

\section{Entrevistadoras}

O presente trabalho faz parte da pesquisa de doutoramento de um dos autores (M.I.N.) que foi responsável pela realização das entrevistas e pelo treinamento de outra entrevistadora. O treinamento consistiu de uma etapa teórica e outra prática feita em campo 19. Primeiramente, ela assistiu à realização de uma série de entrevistas e depois foi supervisionada enquanto executava algumas entrevistas, até se poder concluir que ela estava preparada para realizar a tarefa sozinha. A revisão dos questionários revelou boa qualidade do preenchimento e consistência dos dados.

\section{Coleta de dados}

A coleta dos dados foi feita por meio de entrevista face-a-face, sendo utilizado um questionário padronizado contemplando os aspectos sóciodemográficos, as características de acesso ao preventivo do câncer do colo do útero e o instrumento em avaliação. A mulher convidada era conduzida a uma sala reservada, onde a entrevis- 
tadora explicava brevemente os motivos da pesquisa. Para aquelas que concordaram em participar foi apresentado o Termo de Consentimento Livre e Esclarecido para leitura e assinatura.

O conteúdo de cada bloco era explicado antes de iniciar as perguntas do mesmo. Um cartão contendo as quatro possibilidades de resposta (nunca, raramente, algumas vezes e sempre) foi usado durante a coleta de dados desse instrumento com o objetivo de facilitar a escolha da opção que melhor representasse a experiência da respondente com o processo interpessoal em análise 20.

As respostas foram registradas em formulário específico e, posteriormente, submetidas à dupla digitação em um banco de dados.

\section{Caracterização das medidas}

Os oito itens da ESPICMG foram inquiridos usando uma escala do tipo Likert com quatro opções de resposta ordenadas de acordo com: "nunca", "raramente", "algumas vezes" e "sempre", com pontuação dos escores de 1 a 4 pontos, respectivamente. O ítem 8 (frequência de discriminação) foi apurado de modo que o maior escore correspondeu à negação de discriminação. $\mathrm{O}$ somatório dos escores varia de 8 a 32, com escores maiores refletindo crescente percepção de satisfação 13 .

Além do instrumento em validação (ESPICMG), o questionário contém um módulo de características sociodemográficas; dois módulos abordam a experiência das respondentes com o teste de Papanicolaou, enfocando o padrão de adesão recomendado no Brasil 21. Assim, além das características sociodemográficas, a análise descritiva abordou as seguintes variáveis: (i) dispor de seguro saúde ( $\operatorname{sim} /$ não); (ii) ter feito preventivo de câncer do colo do útero na vida ( $\operatorname{sim} /$ não); (iii) ter feito preventivo de câncer do colo do útero nos últimos 3 anos (sim/ não); (iv) ter feito o último preventivo de câncer do colo do útero no SUS (sim/não).

\section{Análise dos dados}

Iniciou-se o processo pela avaliação da estrutura unidimensional proposta por Somkin et al. 13 . Para esse fim foi implementada uma análise de fatores confirmatória (AFC) 22,23 que empregou o estimador Weighted Least Squares Mean and Variance Adjusted (WLSMV) implementada em Mplus 6 (Muthén \& Muthén, Los Angeles, Estados Unidos). Foram utilizadas matrizes de correlação policóricas, adequadas para itens com opções de resposta ordinais 24 .

$\mathrm{O}$ ajuste de modelo foi avaliado por três índices. O índice Root Mean Square Error of Ap- proximation (RMSEA) incorpora uma função de penalidade para lidar com a pouca parcimônia expressa pelos graus de liberdade do modelo 18 . Valores $<0,06$ sugerem um bom ajuste, enquanto os valores $>0,10$ indicam ajuste ruim e que $o$ modelo deva ser rejeitado 22 . Como medidas de ajuste incremental 18,22, os índices Comparative Fit Index (CFI) e Tucker-Lewis Index (TLI) comparam o modelo proposto com um modelo nulo de independência. Ambos variam de 0 a 1, e valores acima de 0,90 indicam ajuste adequado 22,25.

Antecipando um possível mau ajuste do modelo e/ou prevendo plausíveis estruturas dimensionais alternativas, o passo seguinte consistiu na re-exploração da estrutura dimensional do instrumento usando uma Modelagem por Equações Estruturais Exploratórias (MEEE) como proposta por Marsh et al. 26. O procedimento consiste em ajustar uma sequência de modelos exploratórios, mas seguindo os métodos empregados em uma análise de fatores confirmatória (AFC/E). Esse procedimento permite relaxar e efetivamente implementar várias restrições impostas pela análise de fatores exploratória (AFE) "tradicional”, mas permite uma rotação. Na presente análise foi usada a rotação obliqua geomin 23 .

Adicionalmente, foram avaliadas as correlações de resíduos, uma vez que dependências condicionais podem indicar possíveis redundâncias de itens 22 . Esse passo é dado pela avaliação dos índices de modificação (IM) que são baseados na inclusão de parâmetros não previamente considerados. Esses índices refletem uma aproximação do quanto o $\chi^{2}$ do modelo reduziria se determinado parâmetro fosse considerado. Possíveis correlações entre erros de mensuração de itens envolvendo valores de IM $\geq 10$ foram examinadas, assim como as modificações sugeridas pela magnitude das mudanças de parâmetros esperadas. Os valores de mudanças esperadas de parâmetros (MEP) são complementares aos IM e antecipam a intensidade e direção das estimativas com as modificações livremente implementadas nas fases subsequentes da análise 22 . Modelos de dimensionalidades crescentes foram testados utilizando os mesmos índices de ajustes apresentados anteriormente. O significado teórico também foi considerado para avaliar o número e o padrão dos fatores.

O passo seguinte consistiu em re-examinar o melhor modelo identificado pela $\mathrm{AFC} / \mathrm{E}$, agora aplicando uma modelagem do tipo AFC estrita. Além de reavaliar as cargas de fatores e as correlações de erro de mensuração em uma perspectiva congenérica, a sequência também envolveu a avaliação da confiabilidade composta (consistência interna) 27, bem como das validades fatoriais convergente e discriminante 18,22 . A confia- 
bilidade composta $\left(\mathrm{CC} ; \rho_{c c}\right)$ e a variância média extraída (VME; $\rho_{v e}$ ), na qual se embasam as validades fatoriais convergente e divergente, são estimadas para cada fator como funções das cargas fatoriais padronizadas dos itens componentes e dos respectivos erros de mensuração (uniqueness) $22,27,28$. As estimativas de $\rho_{c c}$ variam de 0 a 1 e valores $\geq 0,70$ indicam consistência interna satisfatória 22,28 . A estimativa de $\rho_{v e}$ também varia de 0 a 1 . Um fator apresenta validade convergente se a $\mathrm{VME} \geq 0,50$, o que indica que pelo menos $50 \%$ da variância em uma medida se devem ao traço latente hipotetizado. Ao contrário, a validade fatorial convergente é questionável se a $\mathrm{VME}<$ 0,50 já que a variância devido ao erro de medição é então maior do que a variância devido à construção ${ }^{28}$. Em modelos multidimensionais, a validade fatorial discriminante é afirmada se para um certo fator a raiz quadrada daVME está acima das correlações deste fator com qualquer outro pertencente ao sistema 29 e sem superposições de intervalos de confiança, preferencialmente. Os intervalos de $95 \%$ de confiança (IC95\%) de $\rho_{c c}$, $\rho_{v e} \mathrm{e} \sqrt{\rho_{v e}}$ foram obtidos pelo método de bootstrap com 1.000 replicações 30,31.

Avaliações adicionais de cargas cruzadas de itens e de correlações de resíduos foram realizadas baseadas nos IM e nas MEP, respeitando os mesmos critérios e padrão de qualidade de ajuste previamente apresentados. Todas as análises foram conduzidas no programa Mplus 6.

Finalmente, tendo constatado que a especificação fator-item foi adequada (ausência de cargas cruzadas relevante e ausência de correlações de resíduos) e na possibilidade de validade discriminante ruim, uma última rodada confirmatória (AFC) foi implementada para testar e ajustar a solução mais parcimoniosa. Procedimentos intermediários e índices de qualidade de ajuste foram igualmente considerados para avaliar o modelo.

\section{Aspectos éticos}

O estudo foi aprovado pelos comitês de ética do Hospital Geral de Nova Iguaçu e da instituição coordenadora da pesquisa.

\section{Resultados}

A média de idade das respondentes foi de 44,3 anos, com desvio-padrão de 11,9 anos (amplitude: 19-69), sendo que 4,03\% (IC95\%: 2,2; 5,8) estavam na faixa de 20 a 24 anos e 12,1\% (IC95\%: $9,1 ; 15,0)$ na de 60 a 69 anos. A maioria delas se reportou como não branca (71,4\%; IC95\%: 67,3; $75,5)$, casada $(69,9 \%$; IC95\%: 65,$7 ; 74,1)$ e com ocupação apenas no lar (64,1\%; IC95\%: 59,8; $68,5)$. A média de anos de estudo foi 6,3 com desvio-padrão de 3,6 anos (amplitude: 0-15), sendo que $6,4 \%$ (IC95\%: 4,1; 8,5) das mulheres nunca frequentaram a escola, enquanto 1,3\% (IC95\%: $0,2 ; 2,3)$ tinha grau superior. A análise descritiva do perfil de acesso mostrou que 95,3\% (IC95\%: 93,9; 97,2) das mulheres realizaram o teste de $\mathrm{Pa}-$ panicolaou pelo menos uma vez na vida, $74,8 \%$ (IC95\%: 70,8; 78,7) relatam ter feito nos últimos três anos, com número de exames realizados variando de 1 a 6, sendo que 65,5\% (IC95\%: 61,1; $69,7)$ fizeram o último em unidade de saúde do SUS (Tabela 1).

A distribuição dos itens mostrou que as quatro opções da escala foram usadas para representar a experiência dos respondentes. A média dos escores variou de 2,2 (item 4) a 3,5 (item 5), dados apresentados na Tabela 2.

A AFC não sustentou a estrutura unidimensional original uma vez que a avaliação da qualidade do ajuste do modelo mostrou-se satisfatória apenas para dois dos índices usados (TLI e CFI) apresentados na Tabela 3. Sendo assim, soluções alternativas foram testadas.

Na sequência, uma AFC/E foi implementada para testar possíveis modelos com mais de um fator. Uma solução bi-dimensional sem qualquer correlação de resíduos mostrou-se como sendo a mais parcimoniosa. Conforme mostra a Tabela 4, os itens 1, 2, 3, 4 e 7 compuseram um fator, o qual poderia ser provisoriamente chamado de “comunicação", enquanto os itens 5 e 8 tiveram cargas em outro fator, que poderia corresponder ao "estilo interpessoal". Os índices de qualidade do ajuste mostraram-se adequados, mas a correlação de 0,704 entre os dois fatores pode ser considerada alta. O item 6 mostrou uma carga cruzada no fator 2 , ainda que com estimativa abaixo das demais.

A estrutura bi-dimensional postulada na $\mathrm{AFC} / \mathrm{E}$ foi subsequentemente reavaliada por uma AFC estrita com o item 6 forçado apenas no fator 1 . Os índices de ajuste revelaram-se aceitáveis, exceto, de forma limítrofe, o RMSEA $(0,062)$. As cargas fatoriais mostraram-se acima de 0,60 , sendo a mais alta a do item $6(0,818)$. As estimativas de $\rho_{v e}$ e de $\rho_{c c}$ superaram os pontos de corte de 0,50 e 0,70, respectivamente, indicando validade convergente, bem como consistência interna. Todavia, os fatores mostraram-se excessivamente correlacionados (modelo bi-dimensional 1 na Tabela 5).

À inspeção dos IM, verificou-se que a adição da carga cruzada do item 6 no fator 2 convergeria com melhor qualidade de ajuste em função da redução significativa do $\chi^{2}$ do modelo, correspondente a uma MEP de 0,536. Esse parâmetro 
Características sociodemográficas e de acesso ao teste de Papanicolaou de 472 respondentes da Escala de Satisfação com o Processo Interpessoal de Cuidados Médicos Gerais (ESPICMG).

\begin{tabular}{|c|c|c|}
\hline Características sociodemográficas & $\mathrm{n}$ & $\%$ \\
\hline \multicolumn{3}{|l|}{ Faixa etária (anos) } \\
\hline$<30$ & 58 & 12,3 \\
\hline $30-39$ & 125 & 26,5 \\
\hline $40-49$ & 122 & 25,9 \\
\hline $50-59$ & 110 & 23,3 \\
\hline $60-69$ & 57 & 12,1 \\
\hline \multicolumn{3}{|l|}{ Cor da pele } \\
\hline Branca & 135 & 28,6 \\
\hline Parda & 281 & 59,5 \\
\hline Negra & 56 & 11,9 \\
\hline \multicolumn{3}{|l|}{ Situação conjugal } \\
\hline Casada & 330 & 69,9 \\
\hline Solteira & 23 & 4,9 \\
\hline Divorciada/Separada & 80 & 16,9 \\
\hline Viúva & 39 & 8,3 \\
\hline \multicolumn{3}{|l|}{ Ocupação } \\
\hline Do lar & 301 & 63,8 \\
\hline Outras ocupações & 171 & 36,2 \\
\hline \multicolumn{3}{|l|}{ Anos de estudo } \\
\hline Nenhum & 30 & 6,4 \\
\hline $1-4$ & 142 & 30,1 \\
\hline $5-8$ & 162 & 34,3 \\
\hline $9-11$ & 132 & 27,9 \\
\hline $12 e+$ & 6 & 1,3 \\
\hline \multicolumn{3}{|l|}{ Características de acesso } \\
\hline \multicolumn{3}{|l|}{ Possuir plano de saúde } \\
\hline $\operatorname{Sim}$ & 63 & 13,3 \\
\hline Não & 409 & 86,7 \\
\hline \multicolumn{3}{|l|}{ Teste de Papanicolaou na vida } \\
\hline $\operatorname{Sim}$ & 450 & 95,3 \\
\hline Não & 22 & 4,7 \\
\hline \multicolumn{3}{|c|}{ Teste de Papanicolaou nos últimos 3 anos } \\
\hline Sim & 353 & 74,8 \\
\hline Não & 119 & 25,2 \\
\hline \multicolumn{3}{|l|}{ Último teste de Papanicolaou no SUS } \\
\hline $\operatorname{Sim}$ & 309 & 65,5 \\
\hline Não & 141 & 29,9 \\
\hline Nunca fez & 22 & 4,6 \\
\hline
\end{tabular}

SUS: Sistema Único de Saúde.

foi testado na última etapa da AFC. Houve uma redução do RMSEA para 0,039, mostrando que, nesse aspecto, o modelo melhorou. As cargas permaneceram acima de 0,60 , mas praticamente intercambiáveis com as do Modelo bi-dimensional 1. A carga do item 6 no fator 2 efetivamente estimada foi 0,264 . As estimativas de VME fica- ram abaixo de 0,50 , indicando pouca validade convergente dessa solução; as consistências internas, todavia, permaneceram adequadas (modelo bi-dimensional 2 na Tabela 5). À semelhança do ocorrido no modelo bi-dimensional 1, os fatores aqui também se mostraram fortemente correlacionados. 
Frequência de distribuição das respostas, média e desvio padrão dos escores dos itens da Escala de Satisfação com o Processo Interpessoal de Cuidados Médicos Gerais (ESPICMG).

\begin{tabular}{|c|c|c|c|c|c|}
\hline \multirow[t]{3}{*}{ Item } & \multicolumn{4}{|c|}{ Frequência de respostas } & \multirow[t]{2}{*}{ Escore } \\
\hline & $\begin{array}{l}\text { Nunca } \\
\text { (1) }\end{array}$ & $\begin{array}{l}\text { Raramente } \\
\text { (2) }\end{array}$ & $\begin{array}{l}\text { Algumas vezes } \\
\text { (3) }\end{array}$ & $\begin{array}{l}\text { Sempre } \\
\text { (4) }\end{array}$ & \\
\hline & n (\%) & n (\%) & n (\%) & n (\%) & Média (DP) \\
\hline $\begin{array}{l}\text { 1. Com que frequência os médicos dão para a Sra. } \\
\text { a informação que a Sra. precisa sobre a sua saúde? }\end{array}$ & $60(12,7)$ & $87(18,4)$ & $150(31,8)$ & $175(37,1)$ & $2,9(1,0)$ \\
\hline $\begin{array}{l}\text { 2. Com que frequência os médicos dão tempo } \\
\text { suficiente para a Sra. fazer suas perguntas? }\end{array}$ & $101(21,4)$ & $116(24,6)$ & $122(25,8)$ & $133(28,2)$ & $2,6(1,1)$ \\
\hline $\begin{array}{l}\text { 3. Com que frequência os médicos escutam com } \\
\text { paciência o que a Sra. tem a dizer? }\end{array}$ & $80(17,0)$ & $117(24,8)$ & $122(25,8)$ & $153(32,4)$ & $2,7(1,1)$ \\
\hline $\begin{array}{l}\text { 4. Com que frequência os médicos perguntam se } \\
\text { a Sra. tem alguma dúvida? }\end{array}$ & $208(44,1)$ & $74(15,7)$ & $85(18,0)$ & $105(22,2)$ & $2,2(1,2)$ \\
\hline $\begin{array}{l}\text { 5. Com que frequência os médicos tratam a Sra. com } \\
\text { dignidade? (de maneira respeitosa/com privacidade). }\end{array}$ & $15(3,2)$ & $45(9,5)$ & $102(21,6)$ & $310(65,7)$ & $3,5(0,8)$ \\
\hline $\begin{array}{l}\text { 6. Com que frequência os médicos demonstram } \\
\text { interesse pela Sra.? }\end{array}$ & $54(11,4)$ & $107(22,7)$ & $145(30,7)$ & $166(35,2)$ & $2,9(1,0)$ \\
\hline $\begin{array}{l}\text { 7. Com que frequência os médicos aceitam as ideias } \\
\text { que a Sra. tem sobre seus problemas de saúde? }\end{array}$ & $184(39,0)$ & $87(18,4)$ & $107(22,7)$ & $94(19,9)$ & $2,2(1,2)$ \\
\hline $\begin{array}{l}\text { 8. Com que frequência a Sra. se sente discriminada } \\
\text { por médicos? }\end{array}$ & $56(11,9)$ & $87(18,4)$ & $56(11,9)$ & $273(57,8)$ & $3,1(1,1)$ \\
\hline
\end{tabular}

DP: desvio-padrão.

As estimativas obtidas com a raiz quadrada da VME não sustentaram adequadamente a validade discriminante esperada, sendo os valores inferiores aos das correlações entre os fatores, exceto para o fator 2 do modelo bi-dimensional 1. Neste modelo (modelo bi-dimensional 1), a $\sqrt{\rho_{v e}}$ do fator 1 foi 0,727 (IC95\%: 0,695; 0,759) e a do fator 2 foi 0,807 (IC95\%: 0,754; 0,860). No modelo bi-dimensional 2 , a $\sqrt{\rho_{v e}}$ do fator 1 foi 0,687 (IC95\%: 0,651; 0,723) e a do fator 2 foi 0,691 (IC95\%: 0,647; 0,735).

Os resultados desse processo sequencial implementado com a AFC/E sugeriram a estrutura bi-dimensional como aceitável. A melhoria presumida, com as especificações de cargas cruzadas mostradas na Tabela 4 e projetadas pelos IM e MEP, não foi plenamente corroborada. Desse modo, o modelo bi-dimensional 1 foi o mais parcimonioso, com melhores índices de consistência interna e de validade fatorial convergente, a despeito de ter apresentado fraca validade discriminante.

\section{Discussão}

O instrumento ESPICMG é novo, pouco estudado, possivelmente em fase de desenvolvimento, com espaço para adicionais avaliações. Segundo seu histórico psicométrico, o instrumento tem boa consistência interna e cargas fatoriais satisfatórias, além de, teoricamente, abordar aspectos interpessoais do processo de cuidado considerados modificáveis 13 . Na literatura revisada, não foram identificados estudos de reavaliação do ESPICMG, sabendo-se apenas que ele deriva do modelo conceitual em três dimensões concebido por Stewart et al. 7 .

A primeira questão que se pretendeu responder foi se o constructo "satisfação com o processo interpessoal" avaliado em uma população com características diferentes daquela onde foi originalmente aplicado está sendo adequadamente captado com um instrumento de composição unidimensional. Pelas evidências aqui apresentadas, esse não parece ser o caso.

Com a unidimensionalidade refutada (Tabela 3), o passo seguinte foi analisar o instrumento com a estrutura bi-dimensional identificada. $\mathrm{O}$ modelo bi-dimensional 1, apresentado na Tabela 5, sugere que a dimensão 1 ("comunicação") 
Análise de fatores confirmatória (AFC) da estrutura unidimensional do instrumento Escala de Satisfação com o Processo Interpessoal de Cuidados Médicos Gerais (ESPICMG): cargas fatoriais e erros de mensuração.

\begin{tabular}{|c|c|c|}
\hline Item & $\begin{array}{l}\text { Modelo unidimensional } \\
\qquad \lambda_{i} \\
\text { Cargas padronizadas } \\
\text { (IC95\%) }\end{array}$ & $\begin{array}{c}\text { Resíduo } \\
\boldsymbol{\delta}_{i} \\
\text { Erros de mensuração } \\
\text { (uniqueness) }\end{array}$ \\
\hline $\begin{array}{l}\text { 1. Com que frequência os médicos dão para a Sra. a informação que a Sra. precisa } \\
\text { sobre a sua saúde? }\end{array}$ & $0,669(0,587 ; 0,752)$ & 0,552 \\
\hline $\begin{array}{l}\text { 2. Com que frequência os médicos dão tempo suficiente para a Sra. fazer suas } \\
\text { perguntas? }\end{array}$ & $0,735(0,661 ; 0,807)$ & 0,459 \\
\hline 3. Com que frequência os médicos escutam com paciência o que a Sra. tem a dizer? & $0,735(0,660 ; 0,810)$ & 0,459 \\
\hline 4. Com que frequência os médicos perguntam se a Sra. tem alguma dúvida? & $0,612(0,511 ; 0,712)$ & 0,626 \\
\hline $\begin{array}{l}\text { 5. Com que frequência os médicos tratam a Sra. com dignidade (de maneira respeitosa/ } \\
\text { com privacidade)? }\end{array}$ & $0,741(0,647 ; 0,835)$ & 0,451 \\
\hline 6. Com que frequência os médicos demonstram interesse pela Sra.? & $0,807(0,745 ; 0,869)$ & 0,349 \\
\hline $\begin{array}{l}\text { 7. Com que frequência os médicos aceitam as ideias que a Sra. tem sobre seus } \\
\text { problemas de saúde? }\end{array}$ & $0,747(0,675 ; 0,819)$ & 0,442 \\
\hline 8. Com que frequência a Sra. se sente discriminada por médicos? & $0,665(0,569 ; 0,761)$ & 0,558 \\
\hline RMSEA & 0,089 & \\
\hline $\mathrm{CFI}$ & 0,971 & \\
\hline TLI & 0,960 & \\
\hline
\end{tabular}

CFI: Comparative Fit Index; RMSEA: Root Mean Square Error of Approximation; TLI: Tucker-Lewis Index.

contém itens relacionados ao julgamento da paciente acerca da qualidade da comunicação com os profissionais de saúde, o que é considerado por alguns autores como componente essencial do encontro médico-paciente 1,32,33,34,35. Consistente com outros estudos, o item 1 (informação sobre a saúde) e o item 4 (esclarecimento de dúvidas) dizem respeito à concessão de explicações sobre as condições de saúde 12; o item 2 (tempo para perguntas) e o item 3 (escutar com paciência) correspondem à demonstração de capacidade de resposta 11 (responsiveness) aos problemas da paciente, relacionados à dimensão comunicação.

A dimensão 2 do modelo aborda as atitudes de desrespeito e de discriminação, que também são igualmente valorizadas 36 , parecendo estar alinhada ao "estilo interpessoal" 11. Nesta dimensão são abarcadas, entre outras questões, o interesse pelo paciente como pessoa e a percepção de que o médico é cuidadoso no sentido de que o paciente não se sinta inferior 7, aspectos que são explorados pelo item 5 (dignidade/privacidade) e pelo item 8 (discriminação). A despeito da coerente interpretação e das cargas relativamente altas dos dois itens envolvidos no fator 2 , é importante que se indague sobre o impacto de se ter somente dois indicadores em uma escala.
Essa escassez de itens tende a afetar a estabilidade das propriedades psicométricas 37. Entre outros, pode levar a um insuficiente "mapeamento" do almejado traço latente subjacente, principalmente no que tange à captação de sua crescente intensidade e discriminação. Nesse sentido, a busca de mais itens para compor essa dimensão em estudos futuros seria um passo auspicioso a dar. Vale reparar que a carga cruzada apresentada pelo item 6 e que abarca a "demonstração de interesse do médico pelo paciente” parece já estar indicando um caminho a perseguir e refinar.

Com respeito à dupla carga do item 6 nas dimensões comunicação e estilo interpessoal por ora postuladas, cabem ainda comentários. Primeiro, a falta de diálogo captada pela paciente pode ser decorrente de um tipo de formação profissional que não valoriza a relação pessoal, levando a uma prática pautada por um comportamento impessoal. Uma segunda possibilidade é o médico ter que dar conta da demanda, penalizando outros aspectos essenciais à relação médico-paciente. Segundo Frederiksen et al. 4, esse é um comportamento adotado tendo como referência o modelo biomédico tradicional que pode restringir a qualidade do diálogo, quando captado negativamente pela paciente. Assim, esta carga cruzada acaba se tornando importante 
Análise de fatores confirmatória/exploratória (AFC/E) do instrumento Escala de Satisfação com o Processo Interpessoal de Cuidados Médicos Gerais (ESPICMG): cargas e correlações de fatores.

\begin{tabular}{|c|c|c|c|}
\hline Item & $\begin{array}{c}\text { Fator } 1 \\
\lambda_{i(1)} \\
\text { Cargas padronizadas } \\
\text { (IC95\%) }\end{array}$ & $\begin{array}{c}\text { Fator } 2 \\
\lambda_{i(2)} \\
\text { Erros de mensuração } \\
\quad \text { (uniqueness) }\end{array}$ & $\begin{array}{c}\text { Resíduo } \\
\boldsymbol{\delta}_{\boldsymbol{i}} \\
\text { Correlação de fatores } \\
\text { (IC95\%) }\end{array}$ \\
\hline $\begin{array}{l}\text { 1. Com que frequência os médicos dão para a Sra. a informação que } \\
\text { a Sra. precisa sobre a sua saúde? }\end{array}$ & $0,673(0,543 ; 0,803)$ & $0,014(-0,130 ; 0,147)$ & 0,534 \\
\hline $\begin{array}{l}\text { 2. Com que frequência os médicos dão tempo suficiente para a Sra. } \\
\text { fazer suas perguntas? }\end{array}$ & $0,739(0,637 ; 0,842)$ & $0,013(-0,085 ; 0,111)$ & 0,440 \\
\hline $\begin{array}{l}\text { 3. Com que frequência os médicos escutam com paciência o que a } \\
\text { Sra. tem a dizer? }\end{array}$ & $0,832(0,666 ; 0,998)$ & $-0,092(-0,287 ; 0,104)$ & 0,407 \\
\hline $\begin{array}{l}\text { 4. Com que frequência os médicos perguntam se a Sra. tem alguma } \\
\text { dúvida? }\end{array}$ & $0,707(0,526 ; 0,887)$ & $-0,096(-0,312 ; 0,119)$ & 0,587 \\
\hline $\begin{array}{l}\text { 5. Com que frequência os médicos tratam a Sra. com dignidade (de } \\
\text { maneira respeitosa/com privacidade)? }\end{array}$ & $0,124(-0,219 ; 0,466)$ & $0,728(0,400 ; 1,055)$ & 0,328 \\
\hline 6. Com que frequência os médicos demonstram interesse pela Sra.? & $0,544(0,370 ; 0,717)$ & $0,334(0,151 ; 0,516)$ & 0,338 \\
\hline $\begin{array}{l}\text { 7. Com que frequência os médicos aceitam as ideias que a Sra. tem } \\
\text { sobre seus problemas de saúde? }\end{array}$ & $0,666(0,497 ; 0,836)$ & $0,112(-0,096 ; 0,319)$ & 0,439 \\
\hline 8. Com que frequência a Sra. se sente discriminada por médicos? & $-0,005(-0,020 ; 0,010)$ & $0,797(0,690 ; 0,904)$ & 0,370 \\
\hline$\phi_{1 \leftrightarrow 2}$ [correlação de fatores (IC95\%)] & $0,704(0,558 ; 0,850)$ & & \\
\hline RMSEA & 0,053 & & \\
\hline $\mathrm{CFI}$ & 0,990 & & \\
\hline TLI & 0,991 & & \\
\hline
\end{tabular}

CFI: Comparative Fit Index; RMSEA: Root Mean Square Error of Approximation; TLI: Tucker-Lewis Index.

porque significa que o fenômeno existe no contexto sociocultural brasileiro, embora com conteúdo não independentemente especificado. A retirada do item pode até ser cogitada, mas implica uma modificação que está fora do escopo do presente estudo. Como um importante princípio psicométrico, deve-se revisar o fraseamento dos itens, antes de considerar a exclusão de quaisquer deles 20 .

Quando se implementa a adaptação transcultural de um instrumento, algumas divergências inesperadas podem eventualmente aparecer, por vezes, refletindo a heterogeneidade da população alvo ${ }^{38}$. A despeito do cuidadoso processo que envolveu as etapas iniciais do roteiro em curso 15 , as diferenças surgiram e podem ter sido também decorrentes da interpretação que as respondentes deram aos itens. Segundo Streiner \& Norman 20 , nem todos os itens operam como se pretende, alguns podem confundir o respondente e até mesmo não corresponder àquilo que se pensava que ele corresponderia.

Digno de nota foi também o item 7 , relativo à frequência com que os médicos aceitam as ideias da paciente sobre seus problemas de saúde, ter carregado no fator 1 (“comunicação"). Comentários sobre sua formulação indicam que este busca inquirir sobre o interesse demonstrado pelo médico em elucidar e incorporar nas condutas as atitudes, os valores, as ideias e as crenças da paciente em relação ao processo de cuidado ${ }^{8}$. Na fase de elaboração do modelo conceitual de Stewart et al. 7, foi postulado que o item pertenceria à dimensão "estilo interpessoal", mas não reuniu propriedades psicométricas boas, não tendo, assim, sido aproveitado. Em uma avaliação posterior, os autores assumem que haviam excluído medidas conceitualmente relevantes e recomendam mais avaliações 9 .

A despeito das melhorias de ajuste observadas, o modelo com a inserção da carga cruzada não sustentou adequadamente as relações postuladas. As estimativas de VME ficaram abaixo do padrão admissível, indicando que a variabilidade devida ao erro de mensuração foi maior do que aquela devida ao fator latente. Deste modo o primeiro modelo, sem a carga cruzada, se mostrou mais adequado. 
Tabela 5

Análise de fatores confirmatória (AFC) do instrumento Escala de Satisfação com o Processo Interpessoal de Cuidados Médicos Gerais (ESPICMG): cargas, erros de mensuração, estimativas de confiabilidade e de ajuste geral do modelo.

\begin{tabular}{|c|c|c|c|c|}
\hline \multirow[t]{3}{*}{ Itens } & \multicolumn{2}{|c|}{ Modelo bi-dimensional 1} & \multicolumn{2}{|c|}{ Modelo bi-dimensional 2} \\
\hline & $\lambda_{i}$ & $\boldsymbol{\delta}_{i}$ & $\lambda_{i}$ & $\boldsymbol{\delta}_{i}$ \\
\hline & $\begin{array}{c}\text { Cargas } \\
\text { padronizadas } \\
\text { (IC95\%) }\end{array}$ & $\begin{array}{c}\text { Erros de } \\
\text { mensuração } \\
\text { (uniqueness) }\end{array}$ & $\begin{array}{c}\text { Cargas } \\
\text { padronizadas } \\
\text { (IC95\%) }\end{array}$ & $\begin{array}{c}\text { Erros de } \\
\text { mensuração } \\
\text { (uniqueness) }\end{array}$ \\
\hline \multicolumn{5}{|l|}{ Dimensão 1} \\
\hline $\begin{array}{l}\text { 1. Com que frequência os médicos dão para a Sra. a } \\
\text { informação que a Sra. precisa sobre a sua saúde? }\end{array}$ & $0,676(0,593 ; 0,758)$ & 0,543 & $0,686(0,603 ; 0,768)$ & 0,530 \\
\hline $\begin{array}{l}\text { 2. Com que frequência os médicos dão tempo } \\
\text { suficiente para a Sra. fazer suas perguntas? }\end{array}$ & $0,742(0,667 ; 0,816)$ & 0,450 & $0,752(0,677 ; 0,827)$ & 0,435 \\
\hline $\begin{array}{l}\text { 3. Com que frequência os médicos escutam com } \\
\text { paciência o que a Sra. tem a dizer? }\end{array}$ & $0,741(0,666 ; 0,816)$ & 0,451 & $0,753(0,677 ; 0,828)$ & 0,434 \\
\hline $\begin{array}{l}\text { 4. Com que frequência os médicos perguntam se a } \\
\text { Sra. tem alguma dúvida? }\end{array}$ & $0,618(0,517 ; 0,719)$ & 0,618 & $0,626(0,525 ; 0,728)$ & 0,608 \\
\hline $\begin{array}{l}\text { 6. Com que frequência os médicos demonstram } \\
\text { interesse pela Sra.? }\end{array}$ & $0,818(0,755 ; 0,880)$ & 0,332 & $0,776(0,707 ; 0,845)$ & 0,397 \\
\hline $\begin{array}{l}\text { 7. Com que frequência os médicos aceitam as ideias } \\
\text { que a Sra. tem sobre seus problemas de saúde? }\end{array}$ & $0,752(0,680 ; 0,824)$ & 0,434 & $0,762(0,690 ; 0,834)$ & 0,419 \\
\hline \multicolumn{5}{|l|}{ Dimensão 2} \\
\hline $\begin{array}{l}\text { 5. Com que frequência os médicos tratam a Sra. com } \\
\text { dignidade (de maneira respeitosa/com privacidade)? * }\end{array}$ & $0,855(0,752 ; 0,958)$ & 0,269 & $0,852(0,750 ; 0,953)$ & 0,275 \\
\hline $\begin{array}{l}\text { 6. Com que frequência os médicos demonstram } \\
\text { interesse pela Sra.? ** }\end{array}$ & - & - & $0,264(0,134 ; 0,393)$ & - \\
\hline $\begin{array}{l}\text { 8. Com que frequência a Sra. se sente discriminada } \\
\text { por médicos? * }\end{array}$ & $0.755(0.657 ; 0.854)$ & 0,429 & $0,758(0,660 ; 0,857)$ & 0,425 \\
\hline$\phi_{1 \leftrightarrow 2} \quad$ [correlação de fatores (IC95\%)] & $0,794(0$, & ,866) & $0,736(0$, & $, 820)$ \\
\hline RMSEA & & & & \\
\hline CFI & & & & \\
\hline TLI & & & & \\
\hline \multicolumn{5}{|l|}{$\rho_{v e^{* \star \star}}$} \\
\hline Dimensão 1 (IC95\%) & $0,529(0$, & $, 576)$ & $0,472(0$, & $, 522)$ \\
\hline Dimensão 2 (IC95\%) & $0,651(0$, & ,736) & $0,477(0$, & $, 538)$ \\
\hline \multicolumn{5}{|l|}{$\rho_{c c} \#$} \\
\hline Dimensão 1 (IC95\%) & $0,870(0$, & ,891) & $0,841(0,8$ & ,869) \\
\hline Dimensão 2 (IC95\%) & $0,788(0$, & $, 851)$ & $0,713(0$, & ,771) \\
\hline
\end{tabular}

CFI: Comparative Fit Index; RMSEA: Root Mean Square Error of Approximation; TLI: Tucker-Lewis Index.

* Itens componentes do segundo fator;

** Item com carga cruzada no segundo fator do modelo bi-dimensional 2;

*** Variância média extraída (intervalos de 95\% de confiança obtido pelo método bootstrap - B = 1.000);

\# Consistência interna/confiabilidade composta (intervalos de $95 \%$ de confiança obtidos pelo método bootstrap - B = 1.000).

Cabe destacar que as correlações de fatores permaneceram altas nos modelos testados. Estas estimativas quando ficam acima da raiz quadrada das correlações item-fator extraídas sugerem que os modelos carecem de validade discriminante 22 . Isso sugere que haja correla- ções de erros de itens redundantes ou de itens nos seus fatores que podem não ter sido devidamente especificados. É possível, ainda, que um fator de ordem superior ou um fator geral captado via modelos bi-fatores possam responder melhor por essas relações 22,39,40. 
Entre as limitações do presente estudo, pode-se assinalar que os modelos foram testados e modificados com o uso de um único conjunto de dados, carecendo ainda de estudos de replicação e confirmação. Uma segunda limitação é o fato de a população de estudo ser composta por usuárias de serviço público de saúde, selecionada em um cenário sociocultural de baixa renda e diversa daquela na qual o instrumento foi desenvolvido, o que pode ter contribuído para as diferenças encontradas. Ademais, a falta de itens relativos à dimensão "participação na tomada de decisão" que, em princípio, foi postulada no modelo teórico-conceitual original 7 , mas que também não está sendo representada no ESPICMG, revelou uma lacuna na composição multidimensional do instrumento que não pôde ser preenchida, justificando a realização de futuros estudos desenhados para enfrentar a deficiência de número de itens e o mapeamento adequado de três ou mais dimensões.

A comunicação adequada às demandas do paciente dá oportunidade para negociação e inclusão das preferências dele na tomada de decisão 33. Diferentemente de outras escalas 11,12, também baseadas no modelo de Stewart et al. 7, o ESPICMG não contempla itens explicitamente designados para captar tal propensão. Ou seja, formulados para avaliar o esforço do médico em tornar seu paciente ativo, perguntando sobre a preferência de estar ou não envolvido nas decisões, bem como desejando ouvir opiniões e escolhas a respeito de diferentes tratamentos e possíveis resultados 7 .

Como forças do estudo, pode-se assinalar que ele foi conduzido com o uso de um ferramental metodológico refinado e adequado para estudos de validação. Além disso, o tamanho amostral foi apropriado para conferir precisão às estimativas e o percentual de dados perdidos foi baixo.

O ESPICMG foi projetado para capturar percepções do paciente que podem ajudar a identificar aspectos da relação médico-paciente merecedores de melhoramentos. Seu conteúdo parece específico a questões da relação inter- pessoal que, diferentemente de outras como o nível de conhecimento e de habilidades técnicas do profissional, podem indubitavelmente serem apreciadas pelo paciente. O intrumento tem a vantagem de ser breve e fácil de aplicar e, portanto menos cansativo para os respondentes e entrevistadores. Mas, seus itens foram formulados para obter informações sobre os médicos, embora pareçam pertinentes para quaisquer profissionais que interajam com o paciente, constituindo uma desvantagem em relação ao PIPC 11. Também, foi formulado levando em conta apenas quatro pontos na escala Likert, embora opções com cinco até nove categorias pareçam atender melhor a maioria das situações 20 . A restrição pode ter implicado alguma falta de informação acerca da intensidade com que o paciente experimentou os eventos, uma desvantagem em relação ao IPCDPQ modificado por Schillinger et al. 12, usando cinco opções de resposta. Contudo, com o sentido de melhoria contínua, o ESPICMG se mostra adequado para tornar claras algumas condições que não são rotineiramente registradas, embora associadas à não-aderência às recomendações médicas.

No conhecimento dos autores, esta é a sua primeira avaliação implementada com enfoque confirmatório. A não corroboração da estrutura unidimensional e a pouca validade da bi-dimensional encontradas sugerem a possível existência de um fator de ordem superior que não pôde ser efetivamente testado por questões inerentes ao processo de modelagem à luz dos itens existentes ou mesmo à insuficiência de itens para adequadamente discriminar duas ou mais dimensões. Nesse sentido, ainda não há evidências suficientes para recomendar o uso incondicional do instrumento, seja na forma de um único escore bruto especificado a partir do somatório dos escores de itens componentes, seja em subescalas separadas. Novos estudos requerem ser desenvolvidos para aprofundar as estruturas postuladas, até mesmo com a perspectiva de se identificar e estudar novos indicadores/itens que permitam aprimorar o instrumento atual. 


\section{Resumo}

O objetivo do estudo foi reavaliar a estrutura dimensional da versão brasileira da Escala de Satisfação com o Processo Interpessoal de Cuidados Médicos Gerais, originalmente proposta como unidimensional. A análise de fatores confirmatória (AFC) e análise de fatores exploratória (AFC/E) modelada segundo os princípios da AFC foram usadas para identificar o modelo mais parcimonioso e melhor ajustado. A AFC não corroborou a estrutura unidimensional original. Na sequência, a AFC/E ajustou adequadamente uma estrutura bi-dimensional que foi reavaliada pela $A F C$, evidenciando um modelo sem carga cruzada como mais parcimonioso e com adequados índices de ajuste $(C F I=0,982$ e $T L I=0,988)$, exceto o RMSEA $(0,062)$. Embora, o modelo tenha alcançado a validade convergente, a validade discriminante foi questionável, com a raiz quadrada da variância média extraída do fator 1 abaixo da respectiva correlação de fatores. O estudo sugere que ainda não há evidências suficientes para recomendar o uso imediato do instrumento e que são necessários outros estudos para aprofundar as estruturas postuladas.

Relações Médico-Paciente; Questionários; Estudos de Validação; Análise Fatorial

\section{Colaboradores}

Todos os autores contribuíram para a concepção e desenho do estudo, bem como para a interpretação dos resultados e elaboração e revisão do manuscrito. M. E. Reichenheim foi responsável pela organização do trabalho e conduziu a análise estatística.

\section{Agradecimentos}

Os autores agradecem a Karen Barbosa Couto Pereira pela colaboração na coleta de dados. M. E. Reichenheim foi parcialmente apoiado pelo Conselho Nacional de Desenvolvimento Científico e Tecnológico (CNPq, processo 301221/2009-0).

\section{Referências}

1. Martin-Fernández J, Cura-González MD, GómezGascón T, Fernández-López E, Pajares-Carabajal G, Moreno-Jiménez B. Satisfactión del paciente con la relación con su médico de familia: un estudio con el Patient-Doctor Relationship Questionnaire. Aten Primaria 2010; 42:196-205.

2. Pandhi N, Saultz JW. Patients' perceptions of interpersonal continuity of care. J Am Board Fam Med 2006; 19:390-7.

3. Donahue KE, Ashkin E, Pathman DE. Length of patient-physician relationship and patients' satisfaction and preventive service use in the rural south: a cross-sectional telephone study. BMC Fam Pract 2005; 6:40.

4. Frederiksen HB, Kragstrup J, Dehlholm-Lambertsen G. It's all about recognition! Qualitative study of the value of interpersonal continuity in general practice. BMC Fam Pract 2009; 10:47.

5. Haddad S, Potvin L, Roberge D, Pineault R, Remondin M. Patient perception of quality following a visit to a doctor in a primary care unit. Fam Pract 2000; 17:21-9.
6. Rosenthal GE, Shannon SE. The use of patient perceptions in the evaluation of health-care delivery systems. Med Care 1997; 35(11 Suppl):NS58-68.

7. Stewart AL, Nápoles-Springer AM, Pérez-Stable EJ, Posner SF, Bindman AB, Pinderhughes HL, et al. Interpersonal processes of care in diverse populations. Milbank Q 1999; 77:305-39.

8. Nápoles-Springer AN, Santoyo-Olsson J, O’Brien $\mathrm{H}$, Stewart AL. Using cognitive interviews to develop surveys in diverse populations. Med Care 2006; 44(11 Suppl 3):S21-30.

9. Stewart AL, Nápoles-Springer AM, Gregorich SE, Santoyo-Olsson J. Interpersonal processes of care survey: patient-reported measures for diverse groups. Health Serv Res 2007; 42(3 Pt 1):1235-56.

10. Cooper LA, Beach MC, Johnson RL, Inui TS. Delving below the surface. Understanding how race and ethnicity influence relationships in health care. J Gen Intern Med 2006; 21 Suppl 1:S21-7. 
11. Wong ST, Korenbrot CC, Stewart AL. Consumer assessment of the quality of interpersonal processes of prenatal care among ethnically diverse lowincome women: development of a new measure. Womens Health Issues 2004; 14:118-29.

12. Schillinger D, Bindman A, Wang F, Stewart A, Piette J. Functional health literacy and the quality of physician-patient communication among diabetes patients. Patient Educ Couns 2004; 52:315-23.

13. Somkin CP, McPhee SJ, Nguyen T, Stewart S, Shema SJ, Nguyen B, et al. The effects of access and satisfaction on regular mammogram and Papanicolaou test screening in a multiethnic population. Med Care 2004; 42:914-26.

14. Gouveia GC, Souza WV, Luna CF, Souza-Júnior PRB, Szwarcwald CL. Health care users' satisfaction in Brazil, 2003. Cad Saúde Pública 2005; 21 Suppl 1:S109-18.

15. Nascimento MI, Monteiro GTR. Características de acesso ao preventivo de câncer de colo do útero: três etapas metodológicas da adaptação do instrumento de coleta de informação. Cad Saúde Pública 2010; 26:1096-108.

16. Herdman M, Fox-Rushby J, Badia X. A model of equivalence in the cultural adaptation of HRQoL instruments: the universalist approach. Qual Life Res 1998; 7:323-35.

17. Instituto Nacional de Câncer. Plano de ação para redução da incidência e mortalidade por câncer do colo do útero: sumário executivo. Rio de Janeiro: Instituto Nacional de Câncer; 2010.

18. Kline RB. Principles and practice of structural equation modeling. 2nd Ed. New York: Guilford Press; 2005.

19. Moser CA, Kalton G. Survey methods in social investigation. 2nd Ed. London: Heinemann; 1984.

20. Streiner DL, Norman GR. Health measurement scales: a practical guide to their development and use. New York: Oxford University Press; 2008.

21. Instituto Nacional de Câncer. Inquérito domiciliar sobre comportamentos de risco e morbidade referida de doenças e agravos não-transmissíveis: Brasil, 15 capitais e Distrito Federal, 2002-2003. Rio de Janeiro: Instituto Nacional de Câncer; 2004.

22. Brown TA. Confirmatory factor analysis for applied research. New York: Guilford Press; 2006.

23. Loehlin JC. Latent variable models: an introduction to factor, path, and structural equation analysis. $4^{\text {th }}$ Ed. Mahwah: Lawrence Erlbaum; 2003.

24. Muthén B, Asparouhov T. Latent variable analysis with categorical outcomes: multiple-group and growth modeling in Mplus. Los Angeles: Muthén \& Muthén; 2002. (Mplus Web Notes, 4).

25. Tucker LR, Lewis C. A reliability coefficient for maximum likelihood factor analysis. Psychometrika 1973; 38:1-10.
26. Marsh HW, Muthén B, Asparouhov A, Lüdtke O, Robitzsch A, Morin AJS, et al. Exploratory structural equation modeling, integrating CFA and EFA: application to students'evaluations of university teaching. Struc Equ Modeling 2009; 16:439-76.

27. Raykov T, Shrout P. Reliability of scales with general structure: point and interval estimation using a structural equation modeling approach. Struct Equ Modeling 2002; 9:195-212.

28. Hair JF, Black WC, Babin B, Andersen RE, Tatham RL. Multivariate data analysis. 6th Ed. Englewood: Prentice-Hall; 2007.

29. Fornell C, Larcker DF. Evaluating structural equation models with unobservable variables and measurement error. J Mark Res 1981; 18:39-50.

30. Raykov T. Analytic estimation of standard error and confidence interval for scale reliability. Multivariate Behav Res 2002; 37:89-103.

31. Efron B, Tibshirani R. An introduction to the bootstrap. London: Chapman and Hall; 1993.

32. Marcinowicz L, Konstantynowicz J, Chlabicz S. The patient's view of the acceptability of the primary care in Poland. Int J Qual Health Care 2008; 20:27783.

33. Nápoles-Springer AN, Santoyo J, Houston K, Pérez-Stable EJ, Stewart AL. Patient's perceptions of cultural factors affecting the quality of their medical encounters. Health Expect 2005; 8:4-17.

34. Beck RS, Daughtridge R, Sloane PD. Physicianpatient communication in the primary care office: a systematic review. J Am Board Fam Pract 2002; 15:25-38.

35. Williams S, Weinman J, Dale J. Doctor-patient communication and patient satisfaction: a review. Fam Pract 1998; 15:480-92.

36. Blanchard J, Lurie N. R-E-S-P-E-C-T: patient reports of disrespect in the health care setting and its impact on care. J Fam Pract 2004; 53:721-30.

37. Marsh HW, Hau KT, Balla JR, Grayson D. Is more ever too much? The number of indicators per factor in confirmatory factor analysis. Multivariate Behav Res 1998; 33:181-220.

38. Ramírez M, Ford ME, Stewart AL, Teresi JA. Measurement issues in health disparities research. Health Serv Res 2005; 40(5 Pt 2):1640-57.

39. Reise SP, Morizot J, Hays RD. The role of the bifactor model in resolving dimensionality issues in health outcomes measures. Qual Life Res 2007; 16 Suppl 1:19-31.

40. Chen FF, West SG, Sousa KH. A comparison of bifactor and second-order models of quality of life. Multivariate Behav Res 2006; 41:189-225.

Recebido em 25/Jan/2011

Versão final reapresentada em 27/Jun/2011

Aprovado em 12/Jul/2011 